\title{
Assessment and prognostic analysis of EGFR, HER2, and HER 3 protein expression in surgically resected gastric adenocarcinomas
}

This article was published in the following Dove Press journal:

OncoTargets and Therapy

16 December 2014

Number of times this article has been viewed

\section{Daoqiang Tang' \\ Charles YJ Liu ${ }^{2}$ \\ Danping Shen ${ }^{3}$ \\ Shuqiong Fan ${ }^{2}$ \\ Xinying $\mathrm{Su}^{2}$ \\ Peng $\mathrm{Ye}^{2}$ \\ Paul R Gavine 2 \\ Xiaolu Yin ${ }^{2}$}

'Department of Pathology, Ren Ji Hospital, School of Medicine, Shanghai Jiao Tong University, ${ }^{2}$ Department of Translational Science, Asia and Emerging Market iMed, AstraZeneca R\&D, ${ }^{3}$ Department of General Surgery, Ren Ji Hospital, School of Medicine, Shanghai Jiao Tong University, Shanghai, People's Republic of China
Correspondence: Xiaolu Yin Department of Translational Science, Asia and Emerging Market iMed, AstraZeneca R\&D, 199 Liangjing Road, Shanghai 201203, People's Republic of China Email lucy.yin@astrazeneca.com

Paul Gavine

Department of Translational Science, Asia and Emerging Market iMed, AstraZeneca R\&D, 199 Liangjing Road, Shanghai 20I203, People's Republic of China Email paul.gavine@astrazeneca.com
Aim: To investigate the significance of epidermal growth factor receptor (EGFR), human epidermal growth factor receptor (HER)2, and HER3 expression on survival outcomes in Chinese gastric cancer patients.

Materials and methods: Formalin-fixed, paraffin-embedded specimens from 121 patients who underwent gastrectomy at Shanghai Renji Hospital from 2007-2010 were retrospectively examined. Fluorescence in situ hybridization and immunohistochemistry techniques were used to identify gene amplification and protein overexpression. Correlations between the expression or amplification of HER family genes and clinicopathological parameters were then determined using statistical analysis.

Results: EGFR protein overexpression, an increase in HER 2 copy number and gene amplification, and HER3 protein overexpression were identified in $33.1 \%, 17.4 \%$, and $62.0 \%$ of samples, respectively. Statistical analysis showed a significant association between EGFR expression and tumor invasion depth or tumor stage. HER2 was also shown to be significantly associated with the tumor grade. In addition, EGFR protein overexpression was found to be significantly associated with worse overall survival $(P=0.03)$.

Conclusion: The HER family members showed a high expression in gastric cancer. EGFR protein expression was associated with overall survival.

Keywords: gastric cancer, clinicopathologic significance, immunohistochemistry, fluorescence in situ hybridization

\section{Introduction}

Gastric cancer is one of the most frequently diagnosed cancers and is the leading cause of cancer-related death worldwide. ${ }^{1}$ To date, the prognosis of patients with advanced gastric cancer is still poor even after surgery or radical resection. ${ }^{2}$ Adjuvant systemic therapies, including some new biological agents, have been implemented to improve outcomes. ${ }^{3}$

The type I human epidermal growth factor receptor (HER) family consists of four homologous members: ErbB-1 (epidermal growth factor receptor [EGFR]); ErbB-2 (HER2); ErbB-3 (HER3); and ErbB-4 (HER4). All of these are transmembrane receptor tyrosine kinases consisting of three functional domains: an extracellular ligandbinding domain; a lipophilic transmembrane segment; and a cytoplasmic tyrosine kinase domain. ${ }^{4}$ Different from other HER family members, HER3 lacks a functional cytoplasmic tyrosine kinase domain and participates in intracellular signaling through heterodimerization with other HER family members. ${ }^{5}$ Under physiological conditions, while bound to the ligand, those receptors dimerize and activate downstream signaling 
pathways, which leads to cell differentiation, migration, proliferation, or survival. ${ }^{6}$

Genetic alterations in the HER gene family are shown to be related to tumorigenesis and tumor progression in different types of cancer. ${ }^{7}$ EGFR and HER2 overexpression are considered as prognostic factors in gastric cancer and are currently the targets of several novel biological agents, ${ }^{8}$ while HER3 expression is frequently observed in advanced gastric cancer with poor prognosis. ${ }^{9}$ However, the clinical significance of such overexpression is not fully understood, and previous studies showed conflicting results in the association between overexpression of HER family members and poor prognosis. ${ }^{10,11}$ In the present study, we further explored the protein expression and gene amplification of EGFR, HER2, and HER3 in surgically resected gastric adenocarcinoma from a local Chinese cohort, which could hopefully shed some light on the problem.

\section{Materials and methods Patient samples}

A total of 121 gastric adenocarcinoma tissue samples were randomly collected from patients who underwent total or subtotal gastrectomy at the Shanghai Renji Hospital from 2007-2010. Tissue samples were fixed in 10\% neutral formalin and embedded in paraffin before further investigation. Tumor histological subtype was determined according to Lauren's classification ${ }^{12}$ after review by two pathologists. Each tumor sample was classified according to the tumornode-metastasis classification advocated by the International Union against Cancer. ${ }^{13}$ Follow-up data were available from all patients, who were assessed via phone call at 3 months, 6 months, and 12 months after gastrectomy, and then every 6 months thereafter for 5 years or until death. A total of 68 patients received chemotherapy, including FOLFOX (folinic acid, fluorouracil, and oxaliplatin) (37 cases), paclitaxel (two cases), Chinese medicine (one case), and combination therapy (28 cases). Twenty-three patients did not receive chemotherapy, as they were not able to bear the side effects. Chemotherapy information from the rest of the patients was not available. Tumor specimens were collected after obtaining informed consent from the patients, and the protocol of this study was approved by the ethics committee of the Shanghai Renji Hospital.

\section{Immunohistochemistry}

All tumor sections (thickness $=3-5 \mu \mathrm{m}$ ) were stained as per the manufacturer's protocol (Dako Denmark A/S, Glostrup, Denmark). The following primary antibodies were used: EGFR (M7239 mouse monoclonal antibody; Dako pharmDx ${ }^{\text {TM }}$ Kit; Dako Denmark A/S), HER2 (K5204 mouse monoclonal antibody; Dako Denmark A/S), and HER3 (M7297 mouse monoclonal antibody; Dako Denmark A/S). Positive controls were selected from breast or lung carcinomas, which were stained positive in previous tests. For negative controls, primary antibodies were simply replaced by phosphate-buffered saline. The immunostaining was then scored by two independent pathologists who were blinded to the clinical information. Tumor membranous staining was scored using a four-grade scale $(0,1+, 2+$, or $3+)$, and the intensity of cytoplasmic staining for HER 3 was also carefully determined. The following scoring criteria were used: 0 , if no staining was observed; $1+$, if more than $10 \%$ of the tumor cells had weak staining on the membrane (or cytoplasm for HER3); $2+$, if more than $10 \%$ of the tumor cells had moderate staining on the membrane (or cytoplasm for HER3); and 3+, if more than $10 \%$ of the tumor cells had strong staining on the membrane (or cytoplasm for HER3).

\section{Fluorescence in situ hybridization}

HER2 gene amplification was analyzed by fluorescence in situ hybridization using the Vysis Path Vysion HER2/DNA probe kit (HER2 SpectrumOrange/CEP17 SpectrumGreen, Vysis 30-161060) as per the manufacturer's protocol (Abbot Laboratories, Abbott Park, IL, USA). For each specimen, the numbers of gene signals were counted in 50 nuclei, and the mean HER 2 or CEP17 copy number was calculated by dividing the total number of gene signals by 50 . The HER2/ $C E P 17$ ratio was calculated by dividing the total number of HER 2 signals by the total number of CEP17 signals. Finally, amplification of HER2 was defined as a mean HER2 copy number of $>10$ or as a HER2/CEP17 ratio of $\geq 2.0$.

\section{Criteria for defining biomarker positivity}

For EGFR and HER3, scores of 0 or $1+$ were considered negative, and a score of $2+$ or $3+$ was classified as positive or overexpression. For HER2, an immunohistochemistry (IHC) score of $3+$ or an IHC score of $2+$ with amplification of HER 2 was defined as positive, and the IHC scores of 0 and $1+$ or a score of IHC 2+ without an amplification of HER2 were defined as negative.

\section{Statistical analysis}

Survival curves were estimated using the Kaplan-Meier product-limit method, and significant differences between the survival curves were determined using the log-rank test. Survival analyses were conducted using a Cox proportional 
hazards model. The $\chi^{2}$ test was used to assess the possible association between the overexpression of each HER family receptor and the clinicopathologic parameters. Results were considered statistically significant if $P<0.05$. All statistical analyses were conducted using the SPSS 10.0 statistical software program (IBM Corporation, Armonk, NY, USA).

\section{Results \\ EGFR protein overexpression}

In the 121 samples we examined, EGFR expression results were as follows: grade 0, $62(51.2 \%)$; grade $1+, 19(15.7 \%)$; grade 2+, 19 (15.7\%); and grade 3+, 21 (17.3\%). Forty cases $(33.1 \%)$ were classified as positive for EGFR protein expression and $81(66.9 \%)$ cases as negative. Representative images of EGFR immunostaining are shown in Figure 1A-D.
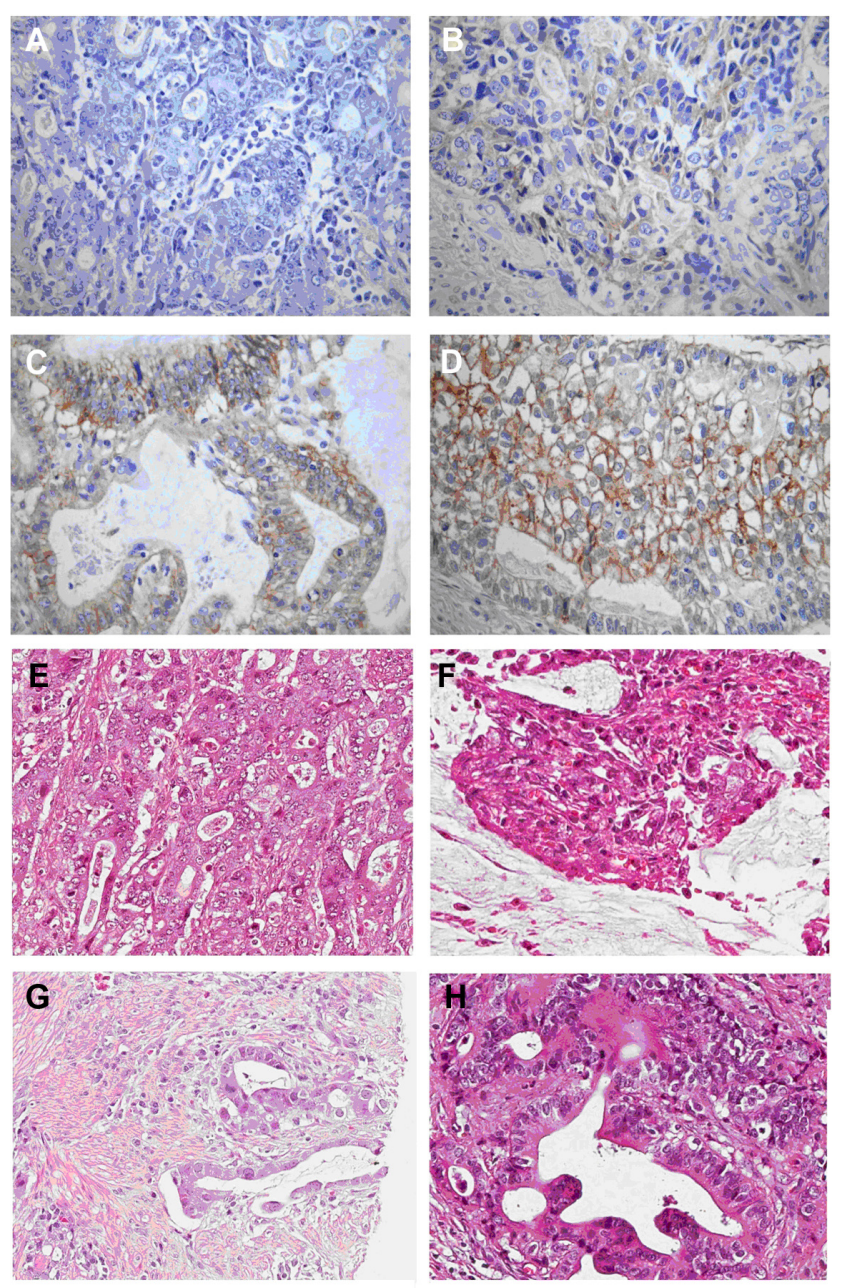

Figure I Representative image showing epidermal growth factor receptor protein expression in gastric cancer samples.

Notes: (A) Immunonegativity; (B) I+ reactivity intensity; (C) 2+ reactivity intensity; (D) 3+ reactivity intensity; $(\mathbf{E}-\mathbf{H})$ representative hematoxylin and eosin staining images for samples in $(\mathbf{A}-\mathbf{D})$, respectively (original magnification $\times 200$ ).
Corresponding hematoxylin and eosin (HE) staining images are shown in Figure 1E-H.

\section{HER2 protein overexpression and gene amplification}

HER2 protein overexpression was observed in $28.1 \%$ of all tumors (16.7\% scored $2+$ and $11.4 \% 3+$ ). A total of $48.2 \%$ of tumors showed totally negative staining (score 0 ), and the remaining $23.7 \%$ showed weak staining (score $1+$ ). HER2 gene amplification was observed in $19(15.7 \%)$ of 121 tumors. HER 2 gene amplification was present in six of 19 tumors with a score of $2+$ and in ten of 19 tumors with a score of $3+$, which was generally consistent with IHC scores. In the 87 samples that exhibited 0 or $1+$ HER2 IHC scores, only three showed HER 2 gene amplification. Twenty-one cases (17.4\%) were classified as positive for HER2 expression and $100(82.6 \%)$ cases as negative. Representative images of HER 2 immunostaining and HER 2 gene amplification are shown in Figure 2A-D and Figure 2E and F, respectively. Corresponding HE staining images for samples in Figure 2A-D are shown in Figure 2G-J.

\section{HER3 protein overexpression}

HER3 protein staining was predominantly located in the cytoplasm. In 115 specimens, HER3 immunostaining results were: grade 0, 18 (15.7\%); grade 1+, 20 (17.4\%); grade 2+, $44(38.3 \%)$; and grade $3+, 32$ (27.8\%). Seventy-six cases $(66.1 \%)$ were classified as positive for HER3 protein expression and $38(33.0 \%)$ as negative. Representative images of HER3 immunostaining are shown in Figure 3A-D. Corresponding HE images are shown in Figure 3E-H.

\section{Coexpression of the HER family members}

The most commonly coexpressed family members were EGFR and HER3, found in $23.1 \%$ of tumors (number [n] $=28$ ). HER 2 and HER 3 coexpression was shown in $11.6 \%$ of tumors $(n=14)$, in which five (4.1\%) also overexpressed EGFR. Only eight tumor samples $(6.6 \%)$ were positive for both EGFR and HER2 expression (Figure 4).

\section{Clinicopathologic parameters and HER expression}

Statistical analysis showed that EGFR overexpression was significantly associated with the depth of tumor invasion and tumor stage (Table 1). HER2 overexpression was also significantly associated with tumor grade, and HER2 positivity 

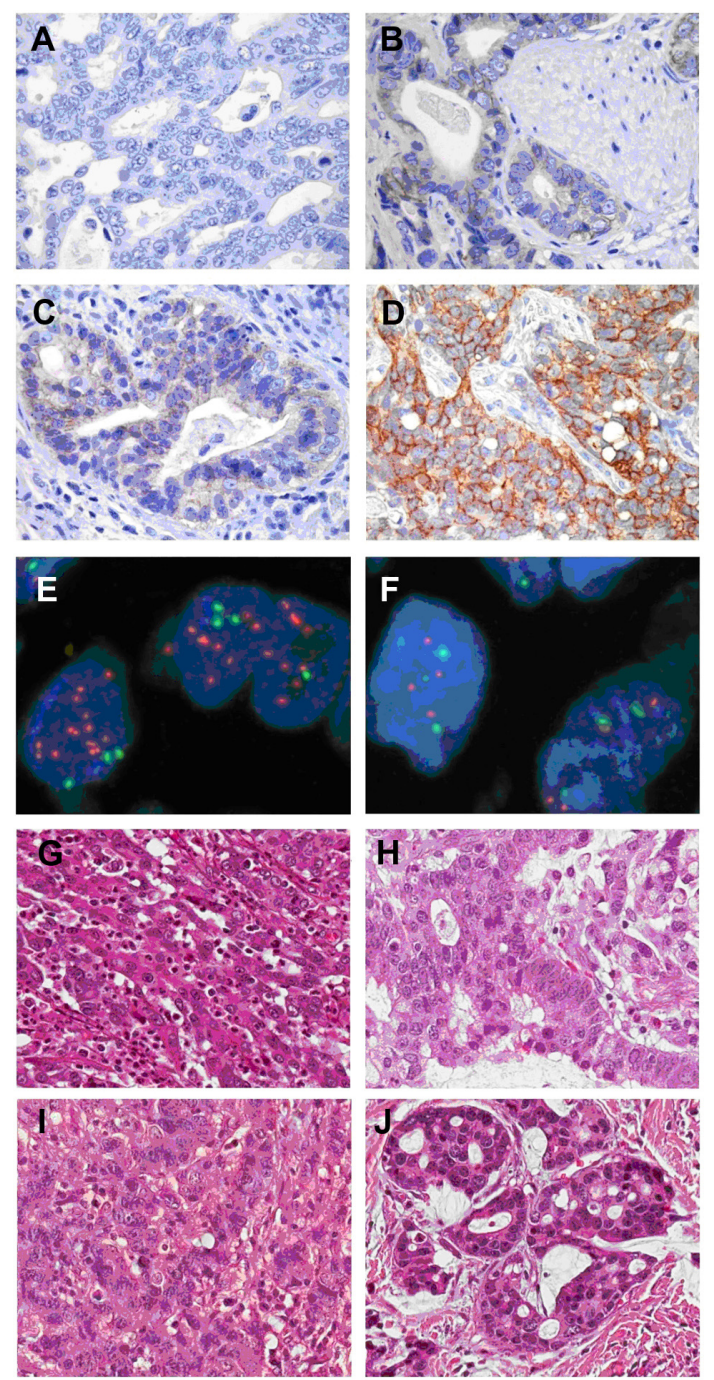

Figure 2 Representative image showing human epidermal growth factor receptor 2 protein expression and gene amplification in gastric cancer samples.

Notes: (A) Immunonegativity; (B) I+ reactivity intensity; (C) 2+ reactivity intensity; (D) 3+ reactivity intensity; (E) FISH positive; (F) FISH negative; (G-J) representative hematoxylin and eosin staining images for samples in (A-D), respectively (original magnification $\times 200$ ).

Abbreviation: FISH, fluorescence in situ hybridization.

was more commonly observed among well- and moderately differentiated types than undifferentiated types $(P=0.0001$; $\chi^{2}$ test). However, no association was shown between HER3 overexpression and sex, age, histological type, or tumornode-metastasis classification.

After standardizing chemotherapy status, a significant relationship was found between EGFR protein overexpression and the overall survival rate $(P=0.03$; Figure $5 \mathrm{~A})$. No significant relationship was shown between the overexpression of other HER family members and overall survival rates (HER2, $P=0.71$; HER $3, P=0.84$; Figure $5 \mathrm{~B}$ and $\mathrm{C}$ ). In addition, overall survival rates were found to be shorter in patients with overexpression of both EGFR and HER3, although with a boundary
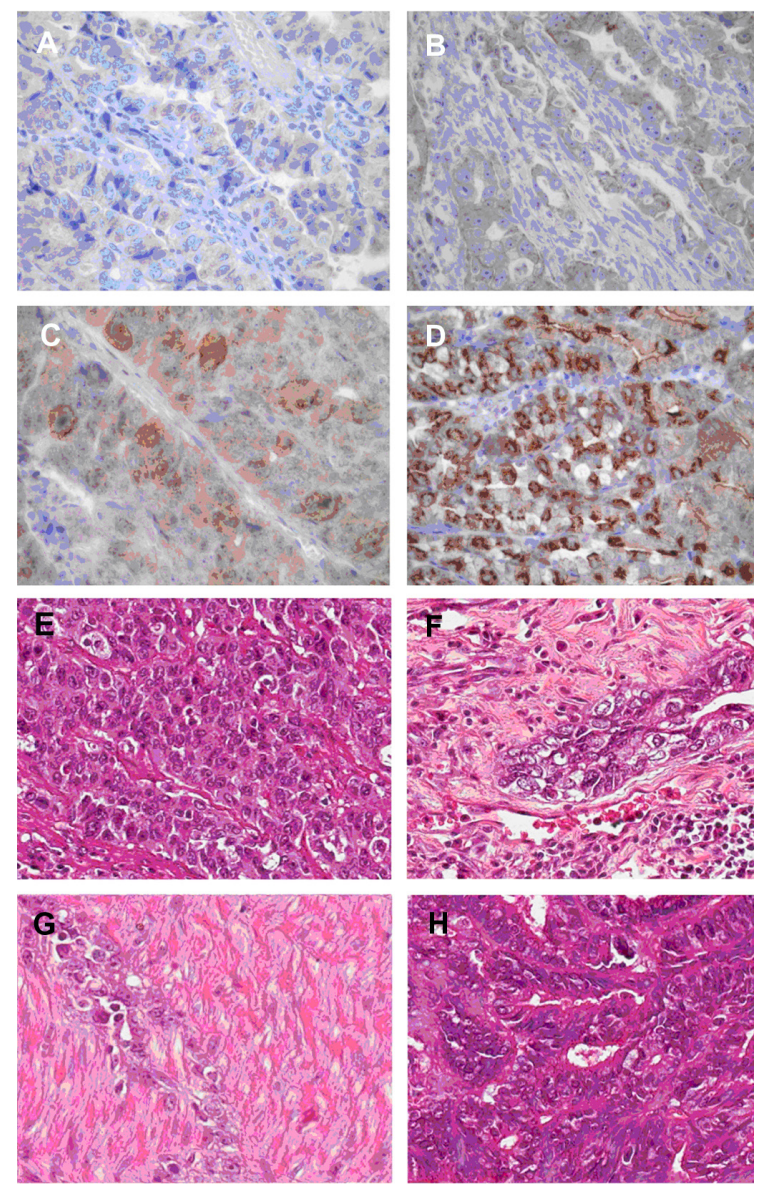

Figure 3 Representative image showing human epidermal growth factor receptor 3 protein expression in gastric cancer samples.

Notes: (A) Immunonegativity; (B) I+ reactivity intensity; (C) 2+ reactivity intensity; (D) 3+ reactivity intensity; $(\mathbf{E}-\mathbf{H})$ representative hematoxylin and eosin staining images for samples in $(\mathbf{A}-\mathbf{D})$, respectively (original magnification $\times 200$ ).

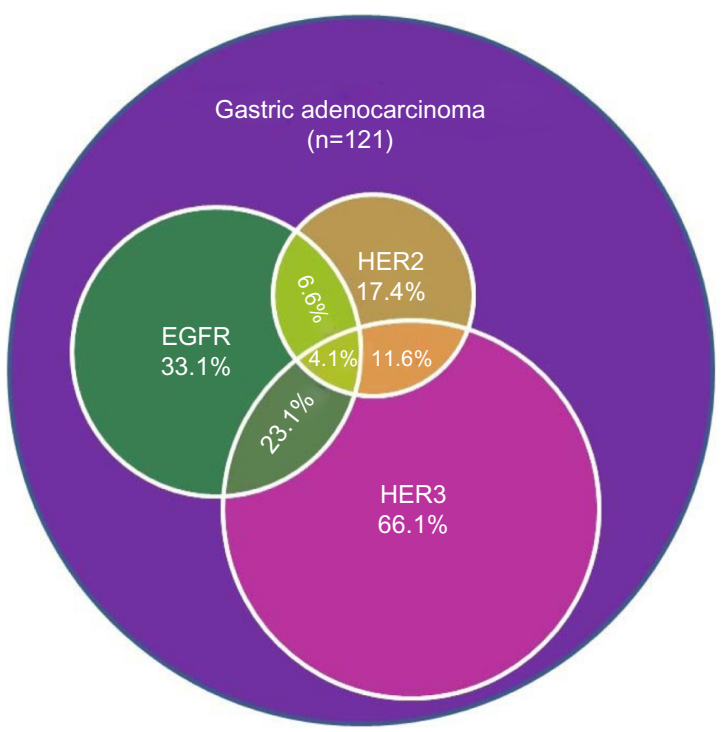

Figure 4 An illustration of the coexpression of HER family members in gastric adenocarcinoma.

Abbreviations: $n$, number; HER, human epidermal growth factor receptor; EGFR, epidermal growth factor receptor. 
Table I Association between clinicopathological parameters and EGFR, HER2, and HER3 expression in I2I cases of gastric cancer

\begin{tabular}{|c|c|c|c|c|c|c|c|}
\hline Parameters & $\mathbf{n}$ & EGFR-positive (\%) & $P$-value & HER2-positive (\%) & $P$-value & HER3-positive (\%) & $P$-value \\
\hline $\mathrm{N}$ & 121 & 40 (33.1\%) & & 21 (17.4\%) & & $76(66.1 \%)$ & \\
\hline \multicolumn{8}{|l|}{ Sex } \\
\hline Male & 85 & 28 (32.9\%) & 0.8062 & $16(22.9 \%)$ & 0.1971 & 52 (65.8\%) & 0.6439 \\
\hline Female & 36 & $12(33.3 \%)$ & & $5(15.6 \%)$ & & $23(67.6 \%)$ & \\
\hline \multicolumn{8}{|l|}{ Tumor grade } \\
\hline II & 23 & $6(26.1 \%)$ & 0.439 & II (55.0\%) & 0.0001 & I8 (78.3\%) & 0.285 \\
\hline III & 87 & 30 (34.5\%) & & $10(13.7 \%)$ & & $53(64.6 \%)$ & \\
\hline IV & 11 & $3(27.3 \%)$ & & $0(0 \%)$ & & $5(62.5 \%)$ & \\
\hline \multicolumn{8}{|l|}{ Tumor stage } \\
\hline I and II & 36 & $6(16.7 \%)$ & 0.0146 & $5(15.6 \%)$ & 0.2689 & $23(65.7 \%)$ & 0.9817 \\
\hline III & 61 & $23(37.7 \%)$ & & 14 (28.6\%) & & 37 (67.3\%) & \\
\hline IV & 24 & II (45.8\%) & & $2(9.5 \%)$ & & 15 (65.2\%) & \\
\hline \multicolumn{8}{|l|}{ Depth of invasion } \\
\hline $\mathrm{T}_{1}$ and $\mathrm{T}_{2}$ & II & $0(0 \%)$ & 0.0001 & $4(40.0 \%)$ & 0.3389 & $8(66.7 \%)$ & 0.6825 \\
\hline $\mathrm{T}_{3}$ and $\mathrm{T}_{4}$ & 110 & 40 (36.4\%) & & 17 (I8.5\%) & & $67(66.3 \%)$ & \\
\hline \multicolumn{8}{|l|}{ Lymph node metastasis } \\
\hline 0 & 31 & $6(19.4 \%)$ & 0.0783 & $3(11.1 \%)$ & 0.1874 & 17 (58.6\%) & 0.3989 \\
\hline 1 & 48 & I5 (3I.3\%) & & $12(30.0 \%)$ & & 32 (7I.I\%) & \\
\hline 2 & 22 & $10(45.5 \%)$ & & $4(23.5 \%)$ & & $12(60.0 \%)$ & \\
\hline 3 & 20 & $9(45.0 \%)$ & & $2(11.1 \%)$ & & 14 (73.7\%) & \\
\hline \multicolumn{8}{|l|}{ Metastasis } \\
\hline Negative & 112 & $36(32.1 \%)$ & 0.8115 & $21(22.1 \%)$ & 0.1049 & $70(67.3 \%)$ & 0.672 \\
\hline Positive & 9 & $4(44.4 \%)$ & & $0(0 \%)$ & & $5(55.6 \%)$ & \\
\hline \multicolumn{8}{|l|}{ Subtype } \\
\hline Intestinal type & 24 & $10(41.7 \%)$ & 0.282 & 9 (37.5\%) & 0.064 & $20(83.3 \%)$ & 0.0859 \\
\hline Diffused type or mixed type & 95 & $28(29.5 \%)$ & & $12(15.4 \%)$ & & $55(63.2 \%)$ & \\
\hline
\end{tabular}

Note: $T_{1}-T_{4}$ is the tumor stage based on tumor-node-metastasis classification advocated by International Union against Cancer.

Abbreviations: EGFR, epidermal growth factor receptor; HER, human epidermal growth factor receptor; n, number.
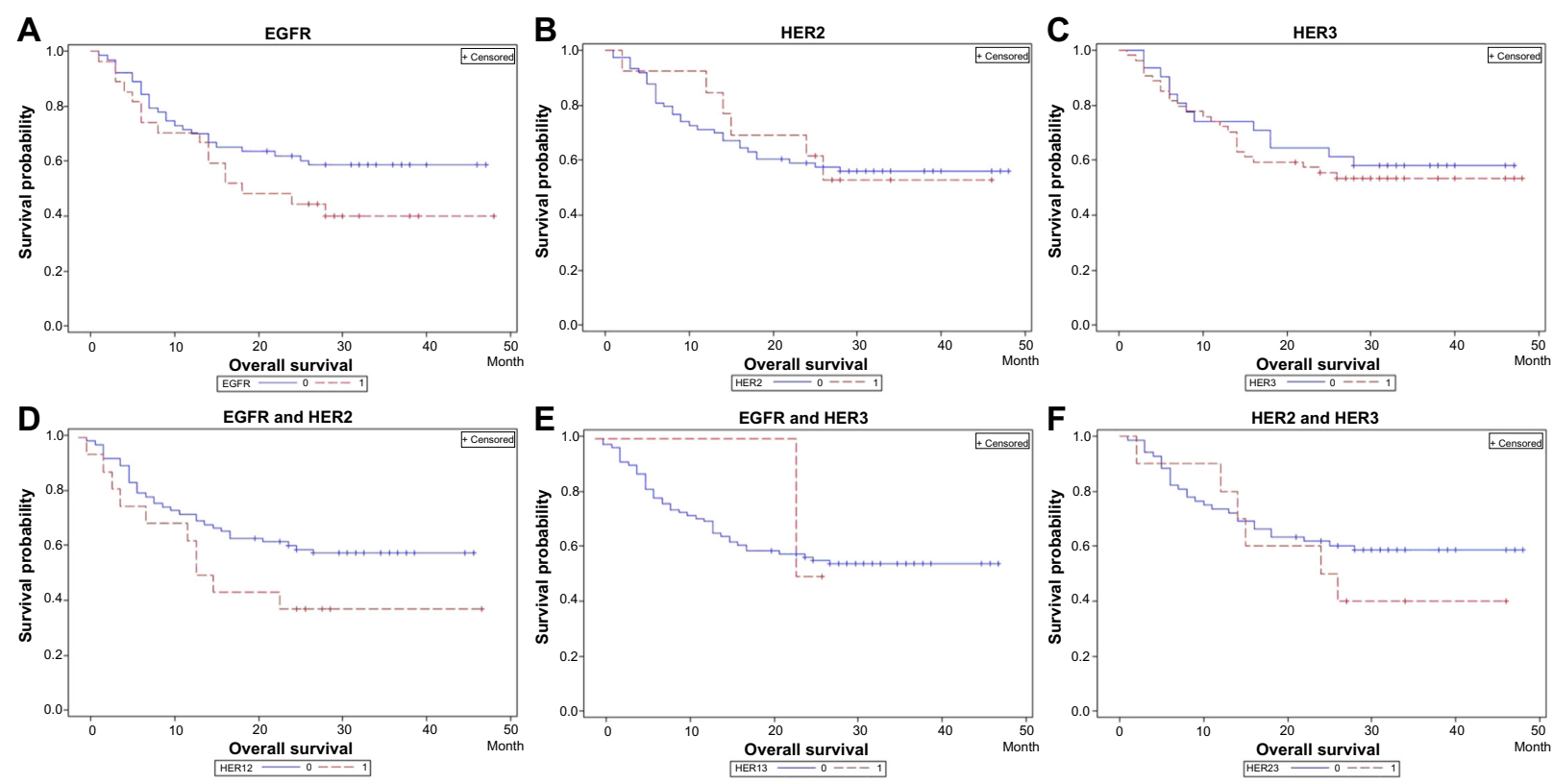

Figure 5 Association between patient overall survival and the overexpression of HER family members.

Notes: (A) EGFR overexpression was significantly associated with worse survival ( $P=0.03$; log-rank test). (B) No significant relationship was found between HER2 and overall survival $(P=0.7 \mathrm{I})$. (C) No significant relationship was found between HER2 and overall survival $(P=0.84)$. (D) Co-overexpression of EGFR and HER3 was related to worse survival, although with a boundary significance $(P=0.07)$. (E) No significant relationship was found between overall survival and co-overexpression of EGFRand HER2 $(P=0.49)$. $(F)$ No significant relationship was found between overall survival and co-overexpression of HER2 and HER3 ( $P=0.98)$. All results were obtained after standardizing chemotherapy status.

Abbreviations: EGFR, epidermal growth factor receptor; HER, human epidermal growth factor receptor. 
significance $(P=0.07$; Figure $5 \mathrm{D})$. No significant association was shown between the expression of other combinations of EGFR, HER2, HER3 and overall survival (EGFR and HER2, $P=0.49$; HER2 and HER3, $P=0.98$; Figure 5E and F).

\section{Discussion}

Overexpression of the HER family members can lead to the activation of downstream signaling pathways, resulting in the cellular transformation and proliferation events associated with tumorigenesis. ${ }^{6}$ HER overexpression in gastric cancer has been reported in many previous studies. However, conclusions regarding the associations between HER positivity and patient survival were conflicting. ${ }^{10,11}$ This study analyzed the expression of the HER family members and assessed their potential influence on patient outcomes. Our study has demonstrated an association between HER overexpression and tumor stage, grade, or overall survival in those gastric cancer patients.

EGFR gene amplification and/or protein expression have been observed in a variety of solid tumors, including lung, colorectal, urinary bladder, breast, head and neck, and esophageal carcinomas. ${ }^{14-19}$ The frequency of EGFR protein expression and/or gene amplification in gastric cancer ranges from $2 \%-35 \% .{ }^{20}$ Possible explanations for this large variation include differences in fixation conditions, antibodies, scoring systems, subjectivity of pathologist interpretation, and intratumoral heterogeneity. In the present study, we detected EGFR positivity in $33.3 \%$ of gastric cancer, which is similar to the findings of previous studies, ${ }^{20,21}$ and we also identified a significant association with the depth of tumor invasion, tumor stage, and overall survival. Indeed, a previous study showed that EGFR protein overexpression was significantly associated with response to cetuximab therapy in metastatic colorectal cancers. ${ }^{22}$ Our study showed more evidence that EGFR positivity could be a predictor of response to EGFRtargeted therapy in gastric cancer.

HER2 expression in gastric cancer was first reported in $1986,{ }^{23}$ which was followed by a large number of related reports. ${ }^{24-27}$ The incidence of HER2 protein expression in gastric cancer varies between studies, ranging from $\sim 9 \%-38 \%{ }^{28}$ In our study, the HER2 protein overexpression and gene amplification was identified in $28.1 \%$ (34/121) and $15.7 \%$ (14/69) of gastric cancers, respectively, leading to a HER2-positive rate of $17.4 \%(21 / 121)$ in patients with gastric cancer. A recent study by $\mathrm{Xu}$ et a ${ }^{29}$ showed a similar positivity rate (11.2\%). Furthermore, the HER2 positivity rate appears to be higher in differentiated-type tumors when compared to undifferentiatedtype tumors, and similar results have been reported by other investigators. ${ }^{8}$ The underlying cause for this selectively high expression of HER2 in differentiated-type tumors remains unclear. The role of HER2 as a prognostic factor in gastric cancer also remains unclear. A recent systematic review assessing the impact of HER2 overexpression on survival found that 20 studies (57\%) reported no difference in overall survival, while two $(6 \%)$ studies showed significantly longer overall survival in patients with HER2 overexpression, and 13 (37\%) found significantly worse overall survival in patients with HER 2 overexpression. ${ }^{30}$ In addition, a recent study by Xu et $\mathrm{al}^{29}$ found a significant relationship between HER 2 positivity and the overall survival rate. Our results, on the other hand, provided further evidence that HER2 status does not influence the outcomes of patients with surgically resected gastric adenocarcinomas, which is in contrast to breast cancer.

Although the tyrosine kinase domain of HER3 is nonfunctional, its dimerization with another HER protein is required for the activation of downstream signaling pathways. ${ }^{5}$ Several studies have shown significant correlations between HER3 overexpression and decreased survival rate in patients with colorectal, breast, laryngeal, and esophageal carcinomas. ${ }^{31-34}$ The prognostic relevance of HER3 expression has yet to be elucidated in gastric cancer. In our study, we observed HER3-positive expression in $68.7 \%$ of patients with gastric cancer, although HER3 protein expression was not shown to be associated with any clinicopathologic factors.

Considering its lack of tyrosine kinase functionality, HER3 has not traditionally been regarded as a therapeutic target. ${ }^{35}$ However, blockade of HER3 in in vitro studies downregulated downstream signaling and caused tumor cell death. ${ }^{36}$ In breast cancer, the formation of the HER2-HER3 heterodimer is associated with a significantly decreased survival rate in patients, and the prevention of dimerization has already shown clinical benefits. ${ }^{37}$ Hayashi et $\mathrm{al}^{9}$ have previously inferred that coexpression of the HER family members may have synergistic effects on the progression of gastric cancer. Our data identified the EGFR-HER3 and HER2-HER3 dimers as more frequent expression partners, although no significant correlations were found between the coexpression of the HER family members and clinical outcomes. HER3 blocking therapies, as well as anti-EGFR or anti-HER2 treatment, might therefore represent a viable novel therapeutic strategy for gastric cancer.

\section{Conclusion}

In conclusion, the current study provides further evidence that EGFR overexpression is significantly associated with overall survival in surgically resected gastric adenocarcinomas, 
suggesting that EGFR could be a predictor of response to EGFR-targeted therapy in gastric cancer. Although HER2 or HER3 status are not associated with overall survival in this patient cohort, our data demonstrate the frequent overexpression of HER 3 protein in gastric cancer, and suggest that HER3-targeted agents could represent promising therapeutics for improving gastric cancer patient outcomes. Limitations of our study include the relatively small sample size, which may not have enough statistical power. Studies with larger sample sizes are needed to further clarify the relationship between the HER family and patient prognosis.

\section{Acknowledgments}

We thank Yan Ji for the statistical analysis. This study was sponsored by AstraZeneca.

\section{Disclosure}

The authors affiliated with AstraZeneca are full-time employees and/or have stock in AstraZeneca. The authors report no other conflicts of interest in this work.

\section{References}

1. Strong VE, D'Amico TA, Kleinberg L, Ajani J. Impact of the 7th Edition AJCC staging classification on the NCCN clinical practice guidelines in oncology for gastric and esophageal cancers. J Natl Compr Canc Netw. 2013;11(1):60-66.

2. Zhang XF, Huang CM, Lu HS, et al. Surgical treatment and prognosis of gastric cancer in 2,613 patients. World J Gastroenterol. 2004;10(23): 3405-3408.

3. Sakuramoto S, Sasako M, Yamaguchi T, et al; ACTS-GC Group. Adjuvant chemotherapy for gastric cancer with $\mathrm{S}-1$, an oral fluoropyrimidine. N Engl J Med. 2007;357(18):1810-1820.

4. Walker RA. The erbB/HER type 1 tyrosine kinase receptor family. J Pathol. 1998;185(3):234-235.

5. Olayioye MA, Neve RM, Lane HA, Hynes NE. The ErbB signaling network: receptor heterodimerization in development and cancer. EMBOJ. 2000;19(13):3159-3167.

6. Casalini P, Iorio MV, Galmozzi E, Ménard S. Role of HER receptors family in development and differentiation. J Cell Physiol. 2004;200(3): 343-350.

7. Sergina NV, Moasser MM. The HER family and cancer: emerging molecular mechanisms and therapeutic targets. Trends Mol Med. 2007; 13(12):527-534.

8. Yk W, Cf G, T Y, et al. Assessment of ERBB2 and EGFR gene amplification and protein expression in gastric carcinoma by immunohistochemistry and fluorescence in situ hybridization. Mol Cytogenet. 2011;4(1):14.

9. Hayashi M, Inokuchi M, Takagi Y, et al. High expression of HER3 is associated with a decreased survival in gastric cancer. Clin Cancer Res. 2008;14(23):7843-7849.

10. Begnami MD, Fukuda E, Fregnani JH, et al. Prognostic implications of altered human epidermal growth factor receptors (HERs) in gastric carcinomas: HER2 and HER3 are predictors of poor outcome. J Clin Oncol. 2011;29(22):3030-3036.

11. Terashima M, Kitada K, Ochiai A, et al. Impact of expression of human epidermal growth factor receptors EGFR and ERBB2 on survival in stage II/III gastric cancer. Clin Cancer Res. 2012;18(21): 5992-6000.
12. Laurén, P. The two histological main types of gastric carcinoma: Diffuse and so-called intestinal-type carcinoma. An attempt at a histo-clinical classification. Acta Pathol Microbiol Scand. 1965;64:31-49.

13. Ahn HS, Lee HJ, Hahn S, et al. Evaluation of the seventh American Joint Committee on Cancer/International Union Against Cancer Classification of gastric adenocarcinoma in comparison with the sixth classification. Cancer. 2010;116(24):5592-5598.

14. Robinson KW, Sandler AB. EGFR tyrosine kinase inhibitors: difference in efficacy and resistance. Curr Oncol Rep. 2013;15(4):396-404.

15. Kearsley JH, Leonard JH, Walsh MD, Wright GR. A comparison of epidermal growth factor receptor (EGFR) and c-erbB-2 oncogene expression in head and neck squamous cell carcinomas. Pathology. 1991;23(3):189-194.

16. Bonin S, Lissiani A, Maffezzini M, Stanta G. EGFr and c-erB2mRNA expression in urinary bladder superficial carcinomas. Adv Clin Path. 1997;1(2):155-159.

17. Overman MJ, Hoff PM. EGFR-targeted therapies in colorectal cancer. Dis Colon Rectum. 2007;50(8):1259-1270.

18. Vranic S, Tawfik O, Palazzo J, et al. EGFR and HER-2/neu expression in invasive apocrine carcinoma of the breast. Mod Pathol. 2010;23(5): 644-653.

19. Yoshida K, Kyo E, Tsuda T, et al. EGF and TGF-alpha, the ligands of hyperproduced EGFR in human esophageal carcinoma cells, act as autocrine growth factors. Int J Cancer. 1990;45(1):131-135.

20. Kim MA, Lee HS, Lee HE, Jeon YK, Yang HK, Kim WH. EGFR in gastric carcinomas: prognostic significance of protein overexpression and high gene copy number. Histopathology. 2008;52(6):738-746.

21. Nagatsuma AK, Aizawa M, Kuwata T, et al. Expression profiles of HER2, EGFR, MET and FGFR2 in a large cohort of patients with gastric adenocarcinoma. Gastric Cancer. Epub 2014 Mar 14.

22. Di Fiore F, Sesboüé R, Michel P, Sabourin JC, Frebourg T. Molecular determinants of anti-EGFR sensitivity and resistance in metastatic colorectal cancer. Br J Cancer. 2010;103(12):1765-1772.

23. Fukushige S, Matsubara K, Yoshida M, et al. Localization of a novel v-erbB-related gene, c-erbB-2, on human chromosome 17 and its amplification in a gastric cancer cell line. Mol Cell Biol. 1986;6(3): 955-958.

24. Gravalos C, Jimeno A. HER2 in gastric cancer: a new prognostic factor and a novel therapeutic target. Ann Oncol. 2008;19(9):1523-1529.

25. García I, Vizoso F, Martín A, et al. Clinical significance of the epidermal growth factor receptor and HER2 receptor in resectable gastric cancer. Ann Surg Oncol. 2003;10(3):234-241.

26. Bang YJ, Van Cutsem E, Feyereislova A, et al. Trastuzumab in combination with chemotherapy versus chemotherapy alone for treatment of HER2-positive advanced gastric or gastro-oesophageal junction cancer (ToGA): a phase 3, open-label, randomised controlled trial. Lancet. 2010;376(9742):687-697.

27. Stern HM. Improving treatment of HER2-positive cancers: opportunities and challenges. Sci Transl Med. 2012;4(127):127rv2.

28. Gravalos C, Jimeno A. HER2 in gastric cancer: a new prognostic factor and a novel therapeutic target. Ann Oncol. 2008;19(9):1523-1529.

29. Xu RH, Oiu MZ, Zhou YX, et al. The relationship between HER2 expression and Lauren classification in Chinese gastric cancer patients. J Clin Oncol. 2014;32(5s):abstract 4065.

30. Chua TC, Merrett ND. Clinicopathologic factors associated with HER2-positive gastric cancer and its impact on survival outcomes - a systematic review. Int J Cancer. 2012;130(12):2845-2856.

31. Lédel F, Hallström M, Ragnhammar P, Öhrling K, Edler D. HER3 expression in patients with primary colorectal cancer and corresponding lymph node metastases related to clinical outcome. Eur J Cancer. 2014;50(3):656-662.

32. Hinohara K, Kobayashi S, Kanauchi H, et al. ErbB receptor tyrosine kinase/NF- $\mathrm{KB}$ signaling controls mammosphere formation in human breast cancer. Proc Natl Acad Sci U S A. 2012;109(17):6584-6589.

33. Wei Q, Sheng L, Shui Y, Hu Q, Nordgren H, Carlsson J. EGFR, HER2, and HER3 expression in laryngeal primary tumors and corresponding metastases. Ann Surg Oncol. 2008;15(4):1193-1201. 
34. Yoon HH, Sukov WR, Shi Q, et al. HER-2/neu gene amplification in relation to expression of HER2 and HER3 proteins in patients with esophageal adenocarcinoma. Cancer. 2014;120(3):415-424.

35. Hsieh AC, Moasser MM. Targeting HER proteins in cancer therapy and the role of the non-target HER3. Br J Cancer. 2007;97(4):453-457.
36. van der Horst EH, Murgia M, Treder M, Ullrich A. Anti-HER-3 MAbs inhibit HER-3-mediated signaling in breast cancer cell lines resistant to anti-HER-2 antibodies. Int J Cancer. 2005;115(4):519-527.

37. Baselga J, Swain SM. Novel anticancer targets: revisiting ERBB2 and discovering ERBB3. Nat Rev Cancer. 2009;9(7):463-475.

\section{Publish your work in this journal}

OncoTargets and Therapy is an international, peer-reviewed, open access journal focusing on the pathological basis of all cancers, potential targets for therapy and treatment protocols employed to improve the management of cancer patients. The journal also focuses on the impact of management programs and new therapeutic agents and protocols on

\section{Dovepress}

patient perspectives such as quality of life, adherence and satisfaction. The manuscript management system is completely online and includes a very quick and fair peer-review system, which is all easy to use. Visit http://www.dovepress.com/testimonials.php to read real quotes from published authors.

\footnotetext{
Submit your manuscript here: http://www.dovepress.com/oncotargets-and-therapy-journal
} 